\title{
Dynamical Properties of the Superposition of Two Finite Trio Coherent States
}

\author{
Salama I. Ali1,2, Ahmed M. Mosallem³ \\ ${ }^{1}$ Mathematics Department, Faculty of Science, Hail University, Hail City, Saudi Arabia \\ ${ }^{2}$ Mathematics Department, Faculty of Science, Al-Azher University, Cairo, Egypt \\ ${ }^{3}$ Department of Mathematics and Theoretical Physics, Nuclear Research Centre, Atomic Energy Authority, Cairo, Egypt \\ Email: salama5laser@yahoo.com
}

How to cite this paper: Ali, S.I. and Mosallem, A.M. (2019) Dynamical Properties of the Superposition of Two Finite Trio Coherent States. Journal of Quantum Information Science, 9, 98-109. https://doi.org/10.4236/jqis.2019.91005

Received: November 1, 2018

Accepted: March 26, 2019

Published: March 29, 2019

Copyright (c) 2019 by author(s) and Scientific Research Publishing Inc. This work is licensed under the Creative Commons Attribution International License (CC BY 4.0).

http://creativecommons.org/licenses/by/4.0/

\begin{abstract}
In this contribution we study a superposition of two finite dimensional trio coherent states (FTCS). The state is regarded as a correlated three-mode state in finite dimensional bases. The framework of Pegg and Barnett formalism, and the phase distribution in addition to the Poissonian distribution are examined. It is shown that the eigenvalue of the difference of the photon number (the $q$-parameter) is responsible for the non-classical phenomenon. Furthermore, the quasi-probability distribution functions (the Wigner and $Q$-functions) are also discussed. In this case and for the Wigner function the non-classical behavior is only reported for the odd values of the $q$-parameter.
\end{abstract}

\section{Keywords}

Entropy Squeezing, Atomic Wehrl Entropy, Marginal Atomic Q-Function

\section{Introduction}

The usual CSs introduced by Glauber [1] are eigenstates of the annihilation operator $\hat{a}$ of the harmonic oscillator. Based on Glauber's work, the even and odd CSs were introduced (Dodonov et al., 1974) [2]. The even (odd) CSs are the symmetric (antisymmetric) combination of the CSs. They are two orthonormalized eigenstates of $\left(\hat{a}^{2}\right)$ the square of the annihilation operator $\hat{a}$ and essentially have two kinds of nonclassical effects: the even CS has a squeezing but no antibunching effect, while the odd CS has an antibunching but no squeezing effect (Hillery, 1987; Xia and Guo, 1989) [3]. The simplest archetype examples of nonclassical states are of course number states, whose experimental realization is however difficult to achieve in the optical regime. Moreover, they share very few of the coherence properties that would be desirable both in practical implemen- 
tations and in fundamental experiments. On the other hand, the coherent state has become a useful and necessary tool for treating ideal boson fields subjected to external pumping sources [1] [4] [5] [6]. Although any quantum state can be described in terms of the coherent states due to their resolution of unity, the coherent states themselves do not show non-classical effects such as antibunching, squeezing, etc. Another way of generalizing the coherent states is the pair coherent states (PCS). They are eigen states of the product of the two annihilation operators of the two modes and the photon number difference of these two modes. They show non-classical effects. Agarwal [7] suggested that the optical pair coherent states (PCS) can be generated via the competition of 4-wave mixing and two-photon absorption in a nonlinear medium. Another scheme has been suggested for generating vibrational pair coherent states via the motion of trapped ions in a two-dimensional trap [8].

Another generalized is the trio-coherent state TCS [9], whose various non-classical effects [10] as well as generation scheme [11] have been investigated, is an eigenstate of the operators $\hat{a} \hat{b} \hat{c}, \hat{n}_{a}-\hat{n}_{c}=\hat{P}$ and $\hat{n}_{b}-\hat{n}_{c}=\hat{Q}$ such that

$$
\begin{gathered}
\hat{a} \hat{b} \hat{c}|\xi, p, q\rangle f=\xi|\xi, p, q\rangle f, \hat{Q}|\xi, p, q\rangle f=q|\xi, p, q\rangle f \\
\text { and } \hat{P}|\xi, p, q\rangle f=p|\xi, p, q\rangle f,
\end{gathered}
$$

where $p$ and $q$ are positive integers with $\xi$ is complex number respectively.

Finite dimensional states have also been introduced. The finite dimensional pair coherent state (FPCS) $|\xi, q\rangle$ has been studied recently as the eigenstate of the pair operators $\left(\hat{a}^{\dagger} \hat{b}+\frac{\zeta^{q+1}\left(\hat{a} \hat{b}^{\dagger}\right)^{q}}{(q !)^{2}}\right)$ and the sum of the photon number operators for the two modes $\left(\hat{Q}=\hat{n}_{a}+\hat{n}_{b}\right)$ [12]. Also the superposition of FPCS and some of its properties have been studied recently [13].

In the present communication we develop this idea and introduce the superposition of the finite dimensional trio coherent state (SFTCS). The correlated three-mode for the FTCS is defined as the eigenstate of the following three operators

$$
\hat{X}=\left(\hat{a}^{\dagger} \hat{b} \hat{c}+\frac{\zeta^{q+1} p !\left(\hat{a} \hat{b}^{\dagger} \hat{c}^{\dagger}\right)^{q}}{(q !)^{2}(p+q) !}\right)
$$

for the three modes, and the sum of the photon number operators for the three modes $\hat{a}^{\dagger} \hat{a}+\hat{c}^{\dagger} \hat{c}=\hat{Q}$ and $\hat{b}^{\dagger} \hat{b}-\hat{c}^{\dagger} \hat{c}=\hat{P}$, namely [14]:

$$
\left.\begin{array}{l}
\left(\hat{a}^{\dagger} \hat{b} \hat{c}+\frac{\zeta^{q+1} p !\left(\hat{a} \hat{b}^{\dagger} \hat{c}^{\dagger}\right)^{q}}{(q !)^{2}(p+q) !}|\zeta, p, q\rangle=\zeta|\zeta, p, q\rangle\right. \\
\left(\hat{a}^{\dagger} \hat{a}+\hat{c}^{\dagger} \hat{c}\right)|\zeta, p, q\rangle=q|\zeta, p, q\rangle \\
\left(\hat{b}^{\dagger} \hat{b}-\hat{c}^{\dagger} \hat{c}\right)|\zeta, p, q\rangle=p|\zeta, p, q\rangle
\end{array}\right\}
$$


where the parameter $\zeta$ is a complex variable while the parameters $q \geq 0$ and $p$ are integers and the operators $\hat{a}, \hat{b}$ and $\hat{c}$ commute pairwise. The state takes the form,

$$
|\zeta, q, p\rangle=N_{q, p} \sum_{n=0}^{q} \zeta^{n} \sqrt{\frac{(q-n) ! p !}{q !(n+p) ! n !}}|q-n, n+p, n\rangle,
$$

in the three mode states $\left|n_{a}, n_{b}, n_{c}\right\rangle=\left|n_{a}\right\rangle \otimes\left|n_{b}\right\rangle \otimes\left|n_{c}\right\rangle$, where $\left|n_{s}\right\rangle$ is the Fock state for the mode $s(s=a, b$ and $c)$ and the normalization constant $N_{q, p}$ is given by

$$
N_{q, p}=\left[\sum_{n=0}^{q}|\zeta|^{2 n} \frac{(q-n) ! p !}{q !(n+p) ! n !}\right]^{\frac{-1}{2}},
$$

the parameter $p$ may be a negative integer, in such case the summation starts from $n=|p|$, because of the appearance of the operators $\hat{a}^{\dagger} \hat{b} \hat{c}$ or $\hat{a} \hat{b}^{\dagger} \hat{c}^{\dagger}$ in this form it may be legitimate to call it a finite dimensional trio coherent state.

In this paper we address the problem of constructing and discussing some non-classical properties of the superposition of two finite dimensional trio coherent states. We shall examine some values of the parameters of the state obtained.

The correlated three-mode Schrodinger-cat states $|\zeta, q, p, \phi\rangle$ are defined as superpositions of three (FTCS) separated in phase by $\phi$ (Gerry and Grobe, 1995) [15]:

$$
|\zeta, q, p, \phi\rangle=N_{q, p}^{\phi}\left(|\zeta, q, p\rangle+e^{i \phi}|-\zeta, q, p\rangle\right),
$$

where the normalization constant $N_{q, p}^{\phi}$ is given by

$$
N_{q, p}^{\phi}=\frac{1}{\sqrt{2}}\left[1+\cos \phi N_{q, p}^{2} \sum_{n=0}^{q}(-1)^{n}|\zeta|^{2 n} \frac{(q-n) ! p !}{q !(n+p) ! n !}\right]^{\frac{-1}{2}}
$$

Now we discuss some statistical properties of these correlated two mode states of Equation (4). The results that we are going to present stem from a new approach to the superposing of the finite dimensional state. Subsequently we shall examine the phase distribution in the framework of Pegg and Barnett formalism, the behavior of the sub-Poissonian distribution, the Wigner function and the $Q$-function of the state (4) are discussed.

\section{Phase Distribution}

In this section we shall look at the phase distribution of the above mentioned state. In the Pegg-Barnett formalism for the phase, a Hermitian phase operator is defined in a finite dimensional state space [16]. They used the fact that, in this finite dimensional Hilbert space, one can define phase states rigorously. The phase operator is then defined as the projection operator on the particular phase state multiplied by the corresponding value of the phase. The main idea of the formalism consists in evaluation of all expectation values of physical variables in a finite dimensional Hilbert space. These give real numbers, which depend pa- 
rametrically on the dimension of the space. Because a complete description of the harmonic oscillator involves an infinite number of states to be taken, a limit is taken only after the physical results are evaluated. This leads to proper limits which correspond to the results obtainable in ordinary quantum mechanics. Here, we study phase properties for the state (4) using this formalism.

The Pegg-Barnett phase distribution $P(\eta)$ is defined [16] as

$$
N_{q, p}^{\phi}=\frac{1}{2 \pi} \sum_{l, m} \rho_{l m} \exp \left[i(l-m)\left(\eta-\eta_{0}\right)\right]
$$

the angle $\eta_{0}$ is the phase reference angle and we take it to be zero. In the case of (4) the phase probability distribution is generalized

$$
\begin{aligned}
& P_{q, p}\left(\theta_{1}, \theta_{2}, \theta_{3}, \zeta\right) \\
& =\frac{\left(N_{q, p}\right)^{2}}{(2 \pi)^{3}}\left\{\left\{\left[\frac{q-j}{2}\right] \sum_{n, m=0}^{2 n+j}\left(\zeta^{*}\right)^{2 m+j} \sqrt{\frac{(q-2 n-j) ! p !(q-2 m-j) ! p !}{q !(2 n+j+p) !(2 n+j) ! q !(2 m+j+p) !(2 m+j) !}}\right.\right. \\
& \quad \cdot \exp i\left[(q-2 n-j) \theta_{1}-(q-2 m-j) \theta_{1}+(2 n+p-j) \theta_{2}\right. \\
& \left.\left.-(2 m+p-j) \theta_{2}+(2 n-j) \theta_{3}-(2 m-j) \theta_{3}\right]\right\}
\end{aligned}
$$

Therefore the phases distribution function can be written as

$$
P_{q, p}(\theta, \zeta)=\frac{\left(N_{q, p}\right)^{2}}{(2 \pi)^{3}}\left\{\sum_{n, m=0}^{\left[\frac{q-j}{2}\right]} \zeta^{2 n+j} \sqrt{\frac{(q-2 n-j) ! p !}{q !(2 n+j+p) !(2 n+j) !}} \exp [i 2 n(\theta)]\right\}^{2}
$$

which is normalized according to $\iiint_{-\pi}^{\pi} P_{\zeta, q, p}\left(\theta_{1}, \theta_{2}, \theta_{3}, \zeta\right) \mathrm{d} \theta_{1} \mathrm{~d} \theta_{2} \mathrm{~d} \theta_{3}=1$. Due to the connection between the three modes, the phase distribution depends on the difference between the phases of the modes. In the figures we plot $P_{\zeta, q, p}(\theta)$ against the angle $\theta=\theta_{2}+\theta_{3}-\theta_{1},-\pi \leq \theta \leq \pi$ for different values of the parameter $q, p$ and $|\zeta|$.

To demonstrate the behavior of the distribution function $P_{q, p}(\theta, \zeta)$ we have plotted Figure 1 for $q=p=2$ and different values of the parameter $\zeta$ adjust $\zeta=3,5$. For the $j=0$ case in the earlier study [13] the distribution function shows Gaussian behavior with one peak centered symmetrically around the center of the base, but in the present contribution the function $P_{q, p}(\theta, \zeta)$ decreases its maximum and the peak splits into two separated identical peaks, both are symmetric with respect to the origin, see Figure 1(a). The maxima for the distribution at $\theta=0$ decrease by increasing of the parameter $\zeta$. In Figure 1(b) we take larger values for the parameter $q(q=0)$ and the same values of $\zeta$. We see that the function $P_{\zeta, q}(\theta, \zeta)$ starts at $P_{\zeta, q}(\theta, \zeta)=0.056,(0.125)$ when $|\zeta|=3$, (5) respectively. The maxima of the phase distribution are increased by increasing of the parameter $\zeta$ (see Figure 1(b)). However this increase turns to a decrease for larger values of $\zeta$. The maximum value for $P_{\zeta, q}(\theta, \zeta)$ shifts 


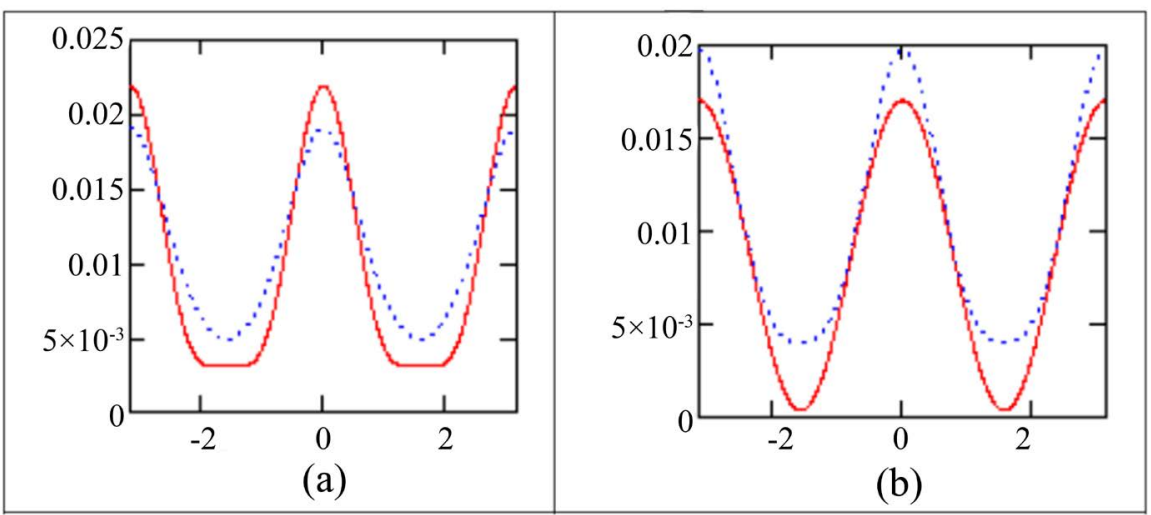

Figure 1. The phase distribution against $\theta$ for fixed values of $p=2, j=0, \zeta=3$ for the solid curve, $\zeta=5$ for the dot curve (a) $-q=2$, (b) $-q=10$.

to higher values of $\zeta$ as $q$ increases. In general the function decreases its height as the $q$-parameter increases. This means that an increase in the value of the $q$-parameter changes the Pegg-Barnett phase distribution function.

\section{Sub-Poissonian Distribution}

In this section we discuss an example of non-classical effects of physical states. One of the non-classical phenomena of the quantized electromagnetic radiation field is the sub-Poissonian statistics. A state of a single mode, for convenience, displaying sub-Poissonian statistics is characterized by the fact that the variance of the photon number $\left\langle\Delta n_{i}\right\rangle^{2}$ is less than the average photon number $\left\langle a_{i}^{\dagger} a_{i}(t)\right\rangle=\left\langle n_{i}\right\rangle$. Sub-Poissonian statistics does not occur in any classical description for the light field, but only occurs when the field is described quaintly [17]. This can be expressed by means of the normalized second-order correlation function as [17]:

$$
g_{z}^{(2)}(\zeta)=\frac{{ }_{j}\left\langle\zeta, p, q\left|\hat{n}_{z}\left(\hat{n}_{z}-1\right)\right| \zeta, p, q\right\rangle_{j}}{{ }_{j}\left\langle\zeta, p, q\left|\hat{n}_{z}\right| \zeta, p, q\right\rangle_{j}^{2}}, \forall z=a, b, c
$$

where

$$
\begin{aligned}
& j_{j}\left\langle\zeta, p, q\left|\hat{n}_{a}\left(\hat{n}_{a}-1\right)\right| \zeta, p, q\right\rangle_{j} \\
& =N_{q, p}^{2} \sum_{n=0}^{\left[\frac{q-j}{2}\right]}|\zeta|^{2(2 n+j)} \frac{(q-2 n-j) ! p !}{q !(2 n+j+p) !(2 n+j) !}(q-2 n-j)(q-2 n-j-1), \\
& \left\langle\zeta, p, q\left|\hat{n}_{b}\left(\hat{n}_{b}-1\right)\right| \zeta, p, q\right\rangle_{j} \\
& =N_{q, p}^{2} \sum_{n=0}^{\left[\frac{q-j}{2}\right]}|\zeta|^{2(2 n+j)} \frac{(q-2 n-j) ! p !}{q !(2 n+j+p) !(2 n+j) !}(p+2 n-j)(p+2 n-j-1), \\
& \left\langle\zeta, p, q\left|\hat{n}_{c}\left(\hat{n}_{c}-1\right)\right| \zeta, p, q\right\rangle_{j} \\
& =N_{q, p}^{2} \sum_{n=0}^{\left[\frac{q-j}{2}\right]}|\zeta|^{2(2 n+j)} \frac{(q-2 n-j) ! p !}{q !(2 n+j+p) !(2 n+j) !}(2 n-j)(2 n-j-1) .
\end{aligned}
$$

and 


$$
\left.\begin{array}{l}
j_{j}\left\langle\zeta, p, q\left|\hat{n}_{c}\right| \zeta, p, q\right\rangle_{j}^{2}=N_{q, p}^{2} \sum_{n=0}^{\left[\frac{q-j}{2}\right]}|\zeta|^{2(2 n+j)} \frac{(q-2 n-j) ! p !}{q !(2 n+j+p) !(2 n+j) !}(q-2 n-j), \\
\left\langle\zeta, p, q\left|\hat{n}_{c}\right| \zeta, p, q\right\rangle_{j}^{2}=N_{q, p}^{2} \sum_{n=0}^{\left[\frac{q-j}{2}\right]}|\zeta|^{2(2 n+j)} \frac{(q-2 n-j) ! p !}{q !(2 n+j+p) !(2 n+j) !}(p+2 n-j), \\
j_{j}\left\langle\zeta, p, q\left|\hat{n}_{c}\right| \zeta, p, q\right\rangle_{j}^{2}=N_{q, p}^{2} \sum_{n=0}^{\left[\frac{q-j}{2}\right]}|\zeta|^{2(2 n+j)} \frac{(q-2 n-j) ! p !}{q !(2 n+j+p) !(2 n+j) !}(2 n-j) .
\end{array}\right\}
$$

where the subscript $z$ relates to the $z$ th mode.

A light field has a sub-Poissonian distribution if $g_{z}^{(2)}<1$, which is a nonclassical effect and means that the probability of detecting an incident pair of photons is less than it would be for a coherent field described by the Poissonian distribution; super-Poissonian distribution if $g_{z}^{(2)}>1$, which is a classical effect $\left(g_{z}^{(2)}=2\right.$ for thermal light) and Poissonian distribution of photons (standard for the coherent state) if $g_{z}^{(2)}=1$.

For fixed the parameter $p=2, j=0$ we examine the correlation function for the first mode $g_{z}^{(2)}(\zeta)$ we have plotted Figure 2. For the first mode the function $g_{z}^{(2)}(\zeta)$ shows sub-Poissonian behavior for all even values of the parameter $q$, however for all range of the parameter $\zeta$ see Figure 2(a). For the second mode and for same values of the parameter $q$ the distribution sub-Poissonian for a short range of $\zeta$. When the parameter $\zeta$ is increased further, the trio-coherent state $|\zeta, q, p\rangle$ exhibits sup-Poissonian behavior. More increases of the variable $\zeta$ the distribution exhibits thermal distribution as well as super-thermal distribution as observed in Figure 2(b). The nonclassical nature of the state is apparent, which $\zeta$ takes on small values shown in Figure 2(b). In the meantime, it is observed that for $q=4$ sub-Poissonian behavior for almost the same range for the cases in which $q=8$. On the other hand the correlation function $g_{z}^{(2)}(\zeta)$ for $q=8$ exhibits thermal distribution faster than the case in which $q=4$. Furthermore, we can also observe that the function starts sub-Poissonian distribution

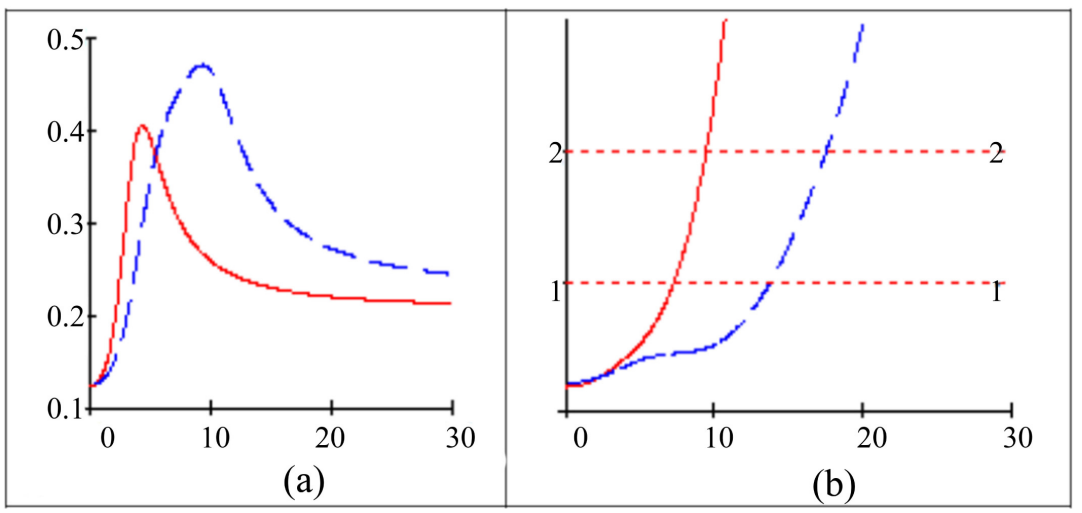

Figure 2. The correlation function $g_{z}^{(2)}(\zeta)$ against the parameter $\zeta$ for fixed value $p=2, X$ (a)-For the first mode $j=0$ and $q=4$ (solid curve), for $q=8$ (dot curve) and for $q=4$ (dash curve). (b)-same as (a) but for the second mode. 
in both modes, but for large values of the parameter $\zeta$ the distribution becomes sub-Poissonian and super thermal states for first and second modes respectively. We note that the behavior of the case $j=1$ for different values of $q$ same as the case $j=0$ with exchanges of the modes.

\section{Quasiprobability Distribution Sub-Poissonian Distribution}

It is well known that there are three quasiprobability distribution functions: $P$-representation, $W$-Wigner, and $Q$-function. These functions are regarded as important tools to provide insight into the nonclassical features of the radiation fields. In the meantime, they have advantages and disadvantages connected with their use. As a part of disadvantage the $P$-function (which describes a quantum state in terms of the probability that the system is in a given coherent state) is highly singular or negative for quantum states with no classical analogues. While the Wigner function may become negative for some quantum states, but it has the considerable advantage for squeezed states that its contour map out the variances in the field quadrature. The $Q$-function is a positive-definite quasiprobability distribution, but its simple relation to anti-normal operator products makes it difficult to interpret in terms of conventional photon counting or squeezing measurements. They are defined by taking the Fourier transforms of their respective characteristic functions:

$$
\begin{aligned}
I(\alpha, \beta, \gamma, s)= & \frac{1}{\pi^{6}} \int_{-\infty}^{\infty} \exp \left(\alpha^{*} \eta-\alpha \eta^{*}\right) \exp \left(\beta^{*} \chi-\beta \chi^{*}\right) \\
& \times \exp \left(\gamma^{*} \xi-\gamma \xi^{*}\right) C(\chi, \eta, \xi, s) \mathrm{d}^{2} \chi \mathrm{d}^{2} \eta \mathrm{d}^{2} \xi
\end{aligned}
$$

where $C(\chi, \eta, \xi, s)$ is the $s$-parameterized characteristic function with the parameter $s=1,0,-1$ corresponding to $P$-representation, Wigner, and $Q$-function, respectively. The characteristic function can be evaluated through the relation

$$
\begin{aligned}
C(\chi, \eta, \xi, s)= & \operatorname{Tr}\left\{\hat{\rho} \exp \left(\hat{b}_{1}^{\dagger} \eta-\hat{b_{1}} \eta^{*}\right) \exp \left(\hat{b}_{2}^{\dagger} \chi-\hat{b}_{2} \chi^{*}\right)\right. \\
& \left.\times \exp \left(\hat{b}_{3}^{\dagger} \xi-\hat{b}_{3} \xi^{*}\right)\left[\frac{s}{2}\left(|\eta|^{2}+|\chi|^{2}+|\xi|^{2}\right)\right]\right\},
\end{aligned}
$$

where $\hat{\rho}$ is the density matrix given by

$$
\hat{\rho}=|q, \zeta, \xi\rangle\langle q, \zeta, \xi|
$$

In what follows we consider the Wigner, and the $Q$-function and for this reason we have to evaluate the integral in Equation (11) for $s=0$ and $s=-1$, respectively. This can be achieved if one manages to calculate the characteristic function. From Equation (12) and after minor algebra we have

$$
\begin{aligned}
& C(\chi, \eta, \xi, s) \\
& =N_{q}^{2} \exp \left[-\frac{1}{2}(1-s)\left(|\eta|^{2}+|\chi|^{2}+|\xi|^{2}\right)\right] \sum_{n=0}^{q} \sum_{r=0}^{q} B_{n} B_{r} L_{n}\left(|\chi|^{2}\right) L_{p+n}\left(|\eta|^{2}\right) L_{q-n}\left(|\xi|^{2}\right)
\end{aligned}
$$




$$
\begin{aligned}
= & \exp \left[-\frac{(1-s)}{2}\left(|\eta|^{2}+|\chi|^{2}+|\xi|^{2}\right)\right]^{\left[\frac{q-j}{2}\right.} \sum_{n=0}^{\frac{q-j}{2}} \sum_{m=0}^{2} B_{n}(\zeta, q) B_{m}^{*}(\zeta, q) \\
& \times \sqrt{\frac{(q-2 n-j) !(p+2 n+j) !(2 n+j) !}{(q-2 m-j) !(p+2 m+j) !(2 m+j) !}} L_{q-2 n-j}^{2 n-2 m}\left(|\chi|^{2}\right) L_{2 n+j}^{2 m-2 n}\left(|\eta|^{2}\right) L_{p+2 n+j}^{2 m-2 n}
\end{aligned}
$$

where $L_{m}^{n}(x)$ are associated Laguerre polynomials and the coefficient $B_{n}(\zeta, q)$ given by

$$
\begin{aligned}
& L_{m}^{n}(x)=\sum_{r=0}^{m}\left(\begin{array}{c}
m+n \\
m-r
\end{array}\right) \frac{(-1)^{r}}{r !} x^{r} \\
& B_{n}(\zeta, q)=\zeta^{2 n+j} \sqrt{\frac{(q-2 n-j) ! p !}{q !(2 n+j+p) !(2 n+j) !}}
\end{aligned}
$$

Having obtained the parameterized characteristic function, we are therefore in a position to find the Wigner, and $Q$-function. This will be evaluated in the next subsections.

\subsection{The Wigner Function}

To obtain the Wigner function $W(\alpha, \beta)$ we insert Equation (14) into Equation (11) and perform the integral. For $s=0$ thus, we obtain

$$
\begin{aligned}
& W(\alpha, \beta)=\frac{4}{\pi^{3}} N_{q}^{2} \exp \left[-2\left(|\eta|^{2}+|\chi|^{2}+|\xi|^{2}\right)\right] \\
& \times \sum_{n=0}^{\left[\frac{q-j}{2}\right]} \sqrt{(2 n+j) !(p+2 n+j) !(q-2 n-j) !(2 r+j) !(p+2 r+j) !(q-2 r-j) !} \\
& \times(-1)^{q+p-2 n-j} \zeta^{2(n+r+j+1)}\left(\frac{\beta \gamma}{\alpha}\right)^{2(r-n)} L_{q-2 n-j}^{2 n-2 r}\left(4|\alpha|^{2}\right) L_{2 n+j}^{2 r-2 n}\left(4|\beta|^{2}\right) L_{p+2 n+j}^{2 r-2 n}\left(|\gamma|^{2}\right)
\end{aligned}
$$

In Figure 3 we have plotted the Wigner function against $\operatorname{Re}(\alpha)$ and $\operatorname{Im}(\alpha)$ for fixed values of $\zeta=5$ and $p=2$, assuming that $\alpha, \beta$ and $\gamma$ are coincidence i.e. in the subspace $\alpha=\beta=\gamma$ [18]. In the meantime we examined the function for the cases in which $j=0$ and $q=0,4,6$ and 8 . When we consider the case in which $q=0$, the function displays Gaussian shape with a symmetrical behavior around zero.

In this case one can see a sharp peak centered at the middle of the base, see Figure 3(a). As soon as we consider the value of the $q$-parameter and take, $j=1$ and $q=1$, the peak of the function gets upsidedown and the nonclassical effect becomes pronounced. This is clear from Figure 3(b) where the negative values of the function are apparent. The spreading of Wigner over the plane is shown as $q$ increases, this is seen for the case in which $j=0$ and $q=6$. In this case the function increases its value and starts with a doubly folded peak in the center towards the positive side. This indicates that the function gets more sensitive to the variation in the $q$-parameter and this of course reflects the change from Gaussian to Fock states; see Figure 3(c). For $j=1$ and $q=5$ the function displays the same shape, however, it changes its direction upsidedown and exhibits 


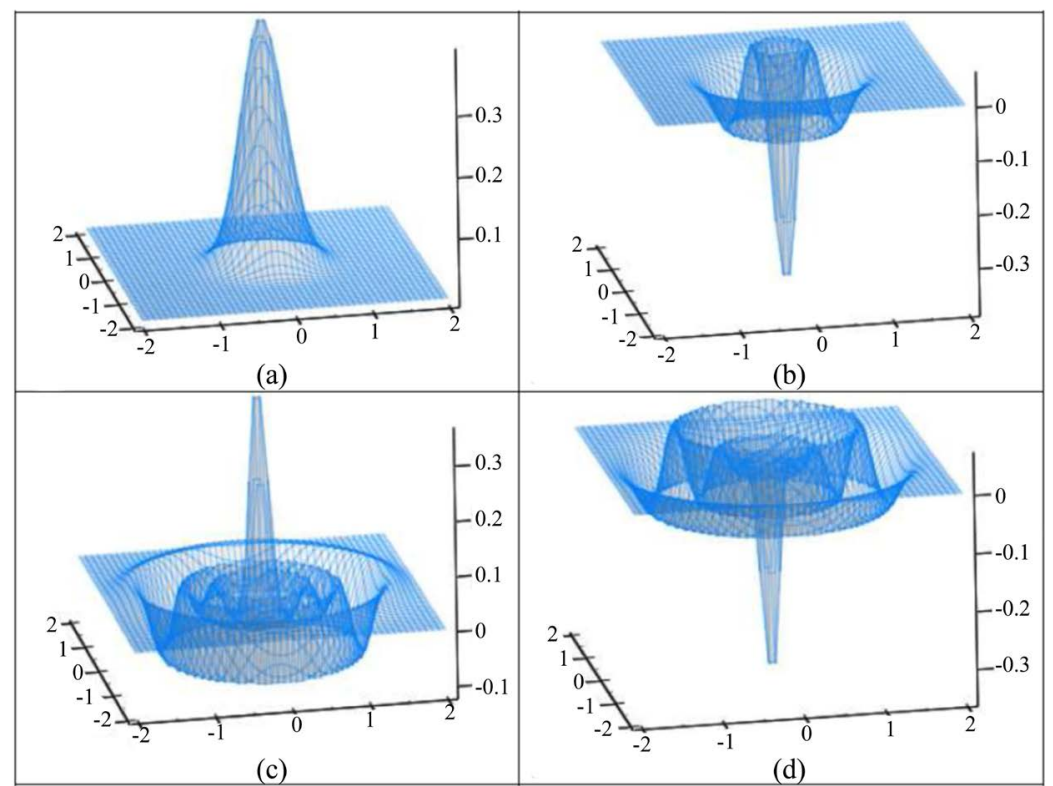

Figure 3. The Wigner function against $\operatorname{Re}(\alpha)$ and $\operatorname{Im}(\alpha)$ for fixed values of $p=2$, $\zeta=5$ and (a) $j=0, q=0$, (b) $j=1, q=1$, (c) $j=0, q=4$, (d) $j=1, q=5$.

negative values. In the meantime, the width of the base increases and the doubly folded peak gets more pronounced, see Figure 3(d). This indicates that the nonclassical behavior appears only for the odd numbers of the $q$-parameter while it disappears for the even numbers. This means that the $q$-parameter plays a role of changing the nonclassical behavior.

\subsection{Q-Function}

In the following we concentrate on one of the quasiprobability distribution functions, that is the $Q$-function [3] [19]. The $Q$-function is a convenient tool to calculate expectation values of anti-normally ordered products of operators. It is well known that the $Q$-function can be defined in terms of diagonal elements of the density operator in the coherent state. Therefore we use the density operator $\rho$ for the state (4) to study its quasi-probability distribution. The $Q$-function in this case is given by

$$
Q(\alpha, \beta, \gamma)=\frac{1}{\pi^{3}}|\langle\alpha, \beta, \gamma \mid \zeta, q, p\rangle|^{2},
$$

where $\alpha, \beta, \gamma \in \mathbb{C}, \mathbb{C}$ is a complex number and $|\alpha, \beta, \gamma\rangle=|\alpha\rangle|\beta\rangle|\gamma\rangle$, with $|\alpha\rangle,|\beta\rangle$ and $|\gamma\rangle$ are the usual coherent states. Generally there are six variables associated with the real and imaginary parts of $\alpha, \beta, \gamma$. For visualization let us confine ourselves to a subspace determined by $\alpha=\beta=\gamma \quad$ [18]. In that subspace the $Q$-function for the state (4) is calculated to be

$$
Q(\alpha, \beta, \gamma)=\frac{\exp \left[-\frac{3}{2}\left(x^{2}+y^{2}\right)\right]}{\pi^{3}}\left|N_{q, p} \sum_{n=0}^{\left[\frac{q-j}{2}\right]} \frac{\zeta^{2 n+j} \alpha^{q+p+2 n+j} \sqrt{p !}}{\sqrt{q !}(p+2 n+j) !(2 n+j) !}\right|^{2},
$$


where $x=\operatorname{Re}(\alpha)$ and $y=\operatorname{Im}(\alpha)$.

Since the maximization or minimization of the $Q$-function depends on the parameter $q$. Therefore, our main task is to examine the behavior of the $Q$-function result of the variation in the $q$-parameter. For this reason we plot Figure 4 for different values of the $q$-parameter keeping all other parameters unchanged as in the Wigner function case. For instance when we consider $j=0$ and $q=0$, the function exhibits Gaussian behavior similar to that of the Wigner function, see Figure 4(a). In this case there exists a unique maximum value at the origin and consequently the function $Q(x, y)$ for the state $|0, \zeta\rangle$ has one peak centered at $(x, y)=(0,0)$. For the case in which $q>0$, more precisely $q=4$, there exists maxima and minima at origin. For the case in which $q=4$, we observe that the function changes its behavior from Gaussian to almost the Fock state. This is quite obvious from the observation of the doubly folded peaks beside an increase in the width of the base, see Figure 4(b). The increase in the value of $q$-parameter leads to an increase in the width of the base, this in addition to a decrease in the maximum value of the function. Thus we may conclude that, the shape of the $Q$-function for the state $|q, \zeta\rangle$ where $q \neq 0$ is sensitive to the change in $q$-parameter where a crater is apparent in the center. However, if we increase $q$ the crater-like at the center spreads out in the phase space and the diameter increases as the $q$.

\section{Conclusion}

In the present paper we have studied the superposition of two entangled finite dimensional trio coherent states. These states can be produced by processes in which there is a strong competition between a three mode parametric conversions. In the mean time, we have employed the Glauber second order-correlation function to examine the nonclassical properties of the state. We have shown that the nonclassical as well as the classical behaviors are apparent in both modes for large values of $\zeta$. However, the nonclassical is more pronounced in the first mode case while the classical behavior is pronounced in the second mode. We have also considered the quasiprobability distribution functions (the Wigner and $Q$-functions) where observation of nonclassical properties is reported for the odd values of the $q$-parameter. In the meantime the $Q$-function displays Gaussian

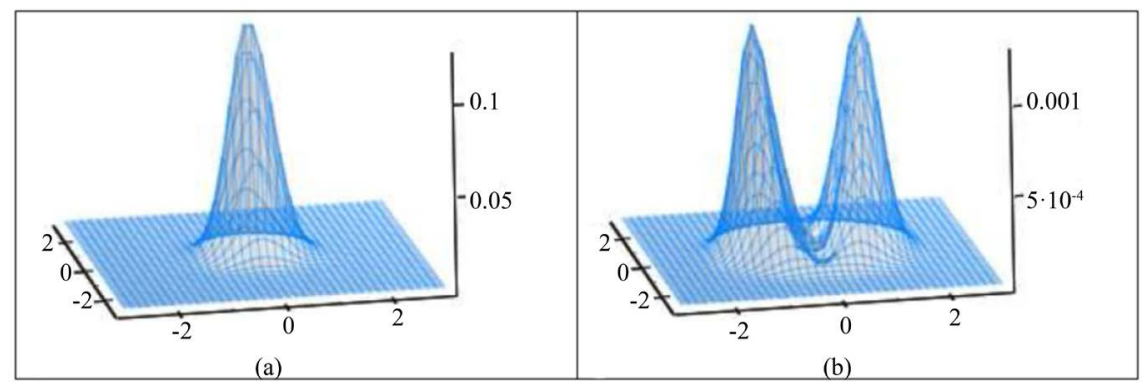

Figure 4. The $Q$-function against $\operatorname{Re}(\alpha)$ and $y=\operatorname{Im}(\alpha)$ for fixed values of $p=2$, $\zeta=5$ and $j=0$ (a) $q=0$, (b) $q=4$. 
behavior and tends to Fock state behavior as $q$ increases. Finally, we have examined the properties of the present state in terms of the phase distribution function introduced by Barnett and Pegg. In this case, regardless of the value of $q$, the function is symmetric about zero. However, as the $q$-parameter increases the value of the function decreases but without broking the symmetry.

\section{Conflicts of Interest}

The authors declare no conflicts of interest regarding the publication of this paper.

\section{References}

[1] Glauber, R.J. (1963) Coherent and Incoherent States of the Radiation Field. Physical Review, 131, 2766-2788. https://doi.org/10.1103/PhysRev.131.2766

[2] Dodonov, V.V., Malkin, I.A. and Manko, V.I. (1974) Even and Odd Coherent States and Excitations of a Singular Oscillator. Physica, 72, 597.

[3] Hillery, M., Connell, R.F.O., Scully, M.O. and Wigner, E.P. (1984) Distribution Functions in Physics: Fundamentals. Physics Reports, 106, 121-167. https://doi.org/10.1016/0370-1573(84)90160-1

[4] Walls, D.F. and Milburn, G.J. (1994) Quantum Optics. Springer, Heidelberg. https://doi.org/10.1007/978-3-642-79504-6

[5] Klauder, J.R. and Sudershan, E.C.G. (1968) Fundamentals of Quantum Optics. Benjamin, New York.

[6] Perlomov, A. (1986) Generalized Coherent States and Their Applications. Springer, Berlin. https://doi.org/10.1007/978-3-642-61629-7

[7] Agarwal, G.S. (1988) Nonclassical Statistics of Fields in Pair Coherent States. Journal of the Optical Society of America B, 5, 1940-1947. https://doi.org/10.1364/JOSAB.5.001940

[8] Gou, S.C., Steinbach, J. and Knight, P.L. (1996) Vibrational Pair Cat States. Physical Review A, 54, 4315-4319. https://doi.org/10.1103/PhysRevA.54.4315

[9] An, N.B. and Duc, T.M. (2002) Trio Coherent States. Journal of Optics B: Quantum and Semiclassical Optics, 4, 80. https://doi.org/10.1088/1464-4266/4/1/313

[10] Obada, A.S.F., Salah, H.H., Darwish, M.A. and Khalil, E.M. (2005) Generalized Trio Coherent States. International Journal of Theoretical Physics, 44, 1347-1364. https://doi.org/10.1007/s10773-005-4770-2

[11] An, N.B. and Duc, T.M. (2002) Generation of Three-Mode Nonclassical Vibrational States of Ions. Physical Review A, 66, Article ID: 065401. https://doi.org/10.1103/PhysRevA.66.065401

[12] Obada, A.S.F. and Khalil, E.M. (2006) Generation and Some Non-Classical Properties of a Finite Dimensional Pair Coherent State. Optics Communications, 260, 19-24. https://doi.org/10.1016/j.optcom.2005.09.080

[13] Khalil, E.M. (2007) Superposition of the Finite Dimensional Pair Coherent State and Some Nonclassical Properties. International Journal of Theoretical Physics, 3 , 9393.

[14] Abdel-Khalek, S., Khalil, E.M. and Ali, S.I. (2008) Entanglement of a in a Finite Trio-Coherent State Two-Level Atom Papered. Laser Physics, 18, 2.

[15] Gerry, C.C. and Grobe, R. (1995) Nonclassical Properties of Correlated Two-Mode 
Schrödinger Cat States. Physical Review A, 51, 1698.

https://doi.org/10.1103/PhysRevA.51.1698

[16] Pegg, D.T. and Barnett, S.M. (1989) Physical Review A, 39, 1665.

[17] Loudon, R. (1983) The Quantum Theory of Light. Clarendon Press, Oxford, 709.

[18] Yi, H., Nguyen, B.A. and Kim, J. (2004) K-Dimensional Trio Coherent States.

[19] Cahill, E. and Glauber, R.J. (1969) Physical Review, 177, 1882. 